\title{
On a Nonlinear Wave Equation with Damping
}

\author{
L. A. Medeiros-M. Milla Miranda
}

Dedicated to Jacques Louis Lions on the occasion of his 60 th birthday.

ABSTRACT. In this work the authors prove the existence of global solutions for damping nonlinear wave equation $u^{\prime \prime}+M\left(\left|A^{\frac{1}{2}} u\right|^{2}\right) A u+A^{\alpha} u^{\prime}=f, 0<\alpha \leq 1$. Uniqueness is obtained for $\frac{1}{2} \leq \alpha \leq 1$. They also prove the exponential decay for the energy, when $0<\alpha \leq 1$.

\section{INTRODUCTION}

In this work we are concerned with global existence and exponential decay for solutions of the mixed problem:

$$
\begin{aligned}
& \frac{\partial^{2} u}{\partial t^{2}}-M\left(\int_{\Omega}|\nabla u(x, t)|^{2} d x\right) \Delta u+(-\Delta)^{\alpha} \frac{\partial u}{\partial t}=f, \text { in } Q, \text { with } 0<\alpha \leq 1 \\
& u(x, t)=0 \text { for }(x, t) \in \Sigma \\
& u(x, 0)=u_{o}(x), \frac{\partial u}{\partial t}(x, 0)=u_{1}(x) \text { in } \Omega,
\end{aligned}
$$

where $M(s)$ is a positive continuous function on $[0, \infty[; \Omega$ is a bounded open set of $\mathbf{R}^{n}$, with smooth boundary $\Gamma$; $Q$ is the cylinder $\left.\Omega \times\right] 0, \infty\left[\right.$ of $\mathbf{R}^{n+1}$, with lateral boundary $\sum=\Gamma \times\left[0, \infty\left[; \Delta=\sum_{i=1}^{n} \partial^{2} \mid \partial x_{j}^{2}\right.\right.$ is the Laplace operator and $|\nabla u(x, t)|^{2}=\sum_{i=1}^{n}\left|\partial u / \partial x_{i}\right|^{2}$.

The equation (1), without damping $(-\Delta)^{\alpha} \frac{\partial u}{\partial t}$, has its origin in the study of vibrations of an elastic string (cf. Carrier [3]). To obtain his model, he

1980 Mathematics Subject Classification (1985 revision): 35L05.

Editorial de la Universidad Complutense. Madrid, 1990. 
admits only vertical component for the tension on the string of length $L$. Using a linear Hooke's law, he obtained the model:

$$
\rho a \frac{\partial^{2} y}{\partial r^{2}}=\left(\tau_{1},+\frac{a E}{2 L} \int_{1}^{l}\left(\frac{\partial u}{\partial x}\right)^{2} d x\right) \frac{\partial^{2} u}{\partial x^{2}}, \quad 0 \leq x \leq L
$$

$\tau_{\sigma}$ is the initial tension on the string; $E$ the Young's modulus of the material; $\rho$ density: $a$ the area of the cross section; $u(x, t)$ the vertical displacement of the point $x$ of the string, at time $t$. In this work we are interested in the case $\tau_{1}>0$, that is. we have an initial tension. If we consider a Hooke's law of the type:

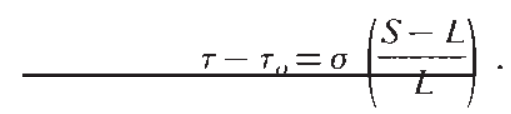

$\sigma$ a function not necessarily linear, $\tau-\tau_{n}$, the variation of the tension, $S-L$ the variation of the lenght of the string, then we obtain a nonlinear model of type ([) . without damping, with

$$
M(s)=m_{1}+\theta(s)
$$

$m_{0}>0 . \theta$ non linear. Therefore the so called Carrier-Narashimham model is:

$$
\frac{\partial^{2} u}{\partial t^{2}}-M\left(\int_{11} \mid \nabla u(x, t)^{2} d x\right) \Delta u=f
$$

When $u_{0}, u_{1}$ are chosen in a regular class of functions, Pohozhaev [17], Lions [7], Arosio-Spagnolo [1] proved that $(*),(1)_{2},(1)_{3}$ has regular solution in $x \in \Omega$, global in $t$, that is, $0 \leq t<\infty$. For the case $n=1$, cf. Bernstein [2], Dickey [4]. Therefore. if we restrict $u_{0} \in H_{o}^{\prime}(\Omega) \cap H^{2}(\Omega), u_{1} \in H_{0}^{1}(\Omega)$,

$f \in L^{\prime}\left(0, T: H_{0}^{\prime}(\Omega)\right)$, no global solutions has been proved to $(*),(1)_{2},(1)_{3}$ and no blow up studied. With this choice for $u_{1}, u_{1}, f$ we can prove that there exists a certain $T_{0}>0$ and a solution for $(*),(1)_{2},(1)_{3}$ defined only on $\Omega \times\left[0, T_{t}\right]$, cf. Ebihara-Medeiros-Milla Miranda [5].

However, with the perturbation $-\Delta \frac{\partial u}{\partial t}$, i.c., the case $\alpha=1$ in $(1)_{1}$, there exist results on global solutions in $t$ and on decay, when $\left(u_{t,}, u_{1}, f\right)$ belongs to $H_{o}^{\prime}(\Omega) \cap H^{2}(\Omega) \times H_{i s}^{\rfloor}(\Omega) \times L^{\prime}\left(0,7 ; H_{o}^{\prime}(\Omega)\right)$, cf. Nishihara [14], Yamada [18]. It is interesting to look Tsutsumi [17].

In the present work, we obtain global solutions of the mixed problem (1), that is, mixed problem for $\left({ }^{*}\right)$ with the damping $(-\Delta)^{\alpha} \cdot \frac{\partial u}{\partial t}$, if $0<\alpha \leq 1$, when $u_{i} \in H_{t}^{l}(\Omega) \cap D\left((-\Delta)^{\alpha}\right), u_{1} \in L^{2}(\Omega), f \in L^{\prime}\left(0, T ; L^{2}(\Omega)\right)$. Our motivation 
was to have information when $\alpha \rightarrow 0$, but no result we know up today. If we suppose $M \in C^{\prime}\left(\left[0, \infty[; \mathbf{R})\right.\right.$ we prove that when $\frac{1}{2} \leq \alpha \leq 1$ we have uniqueness for solutions, of. Theorem 1.2.

In Section 2 we study the exponential decay for solutions obtained in Section $1,0<\alpha \leq 1$, with $f=0$ to avoid technalities, following the ideas of Haraux-Zuazua [6], cf. also Nakao [13], [14], Zuazua [22], Muñoz Rivera [12], Strauss [18]. In Matos-Pereira [10] they obtained algebraic decay for the energy, in the damping case $\alpha=1$, with $M(s)=s$.

Another question, proposed by Lions [7] for $\left({ }^{*}\right),(1)_{2},(1)_{3}$, is to study the case $\left(u_{o}, u_{1}, f\right)$ in $H_{o}^{1}(\Omega) \times L^{2}(\Omega) \times L^{1}\left(0, T ; L^{2}(\Omega)\right)$. An answer to this question was done by Medeiros-Milla Miranda [11]. They proved that the best choice is not the above but the following:

$$
\left(u_{o}, u_{1}, f\right) \in D\left((-\Delta)^{3 / 4}\right) \times D\left((-\Delta)^{1,4}\right) \times L^{1}\left(0, T ; D\left((-\Delta)^{1,4}\right)\right) .
$$

With $D\left((-\Delta)^{\beta}\right)$ one represents the domain of the operator $(-\Delta)^{\beta}$. With this choice the authors proved, in [11], that $(*),(1)_{1},(1)_{2}$ has at least one weak solution, when $\Omega$ is a bounded open set. For the unbounded case of. Matos [9], as an application of the diagonalization theorem by Von NeumannDiximier, cf. Lions-Magenes [8].

The present work is dedicated to Jacques Louis Lions on the occasion of his 60th anniversary, as an acknowledgement of our deep admiration for his scientific work and reconnaissance for his permanent support to our research work.

\section{GLOBAL SOLUTIONS: EXISTENCE AND UNIQUENESS}

Let $V$ and $H$ be real Hilbert spaces with Hilbert structure given, respectively, by $((\cdot, \cdot)),\|\cdot\|,(\cdot, \cdot),|\cdot|$. We suppose $V \subset H$ continuously and $V$ dense in $H$. Let $A$ be the operator defined by the triplet $\{V, H,((\cdot,+))\}$. As it is known, $A$ is a positive self adjoint operator of $H$ with domain $D(A) \subset$ $H$, dense and $D\left(A^{2}\right)=V$. We also know that $((u, u))=(A u, v)$ for all $u \in$ $D(A), v \in \mathrm{V}$ which implies $((u, v))=\left|A^{\frac{1}{2}} u\right|^{2}$. If we suppose $V \subset H$ compact, then the spectral problem $((w, v))=\lambda(w, v)$ for all $\nu \in V$, has for solution a sequence of vectors $\left(w_{v}\right)_{v \in \mathrm{N}}, w_{v} \in H$, for all $v \in \mathbf{N}$, called eigenvectors of $A$ and a sequence $\left(\lambda_{v}\right)_{v \in \mathrm{N}}$ of real numbers $\lambda_{v}$ called eigenvalues of $A$, such that $0<\lambda_{1}<\lambda_{2} \leq \ldots \leq \lambda_{v} \leq \ldots$ diverges to $+\infty$, and $\left(\left(w_{v}, v\right)\right)=\lambda_{v}\left(w_{v}, v\right)$ for all $v \in V, v \in \mathbf{N}$. The sequence $\left(w_{v}\right)_{v \in \mathbf{N}}$ is dense in $V$ and we suppose it is orthonormalized in $H$. 
With this framework, the mixed problem (1) can be written:

$$
\begin{aligned}
& \left.u^{\prime \prime}+M\left(\left|A^{\frac{1}{2}} u\right|^{2}\right) A u+A^{\alpha} u^{\prime}=f \text { in }\right] 0, T[, 0<\alpha \leq 1 \\
& u(0)=u_{o}, \quad u^{\prime}(0)=u_{1}
\end{aligned}
$$

In (2), $u^{\prime}$ denotes the derivative of $u$, with respect to $t$, in the sense of the vector distributions on $] 0, T[$. We assume on $M$ the natural condition:

$$
M \in C^{\prime}\left(\left[0, \infty[; \mathbf{R}), M(s) \geq m_{o}>0 \text { for all } s \geq 0\right.\right.
$$

Theorem 1.1: Suppose $M(s)$ satisfies (3), $0<\alpha \leq 1$,

$$
u_{o} \in V \cap D\left(A^{\alpha}\right), \quad u_{I} \in H, \quad f \in L^{l}(0, T ; H) .
$$

Then, there exists one function $u:[0,7] \rightarrow H, 0<T<\infty$, satisfying the conditions:

$$
\begin{gathered}
u \in L^{\infty}\left(0, T ; V \cap D\left(A^{\alpha}\right)\right) \cap L^{2}\left(0, T ; D\left(A^{\alpha+\frac{1}{2}}\right)\right) \\
u^{\prime} \in L^{\infty}(0, T ; H) \cap L^{2}\left(0, T ; D\left(A^{2}\right)\right) \\
\frac{d}{d t}\left(u^{\prime}(t), v\right)+M\left(\left|A^{\frac{1}{2}} u(t)\right|^{2}\right)\left(A^{2} u(t), A^{\frac{1}{2}} v\right)+\left(A^{\frac{\alpha}{2}} u^{\prime}(t), A^{\frac{\alpha}{2}} v\right)=(f(t), v)
\end{gathered}
$$

in $D^{\prime}(0, T)$ for all $v \in V$. We say $u$ is weak solution of (2).

$$
u(0)=u_{0}, \quad u^{\prime}(0)=u_{l}
$$

Proof: Denote by $V_{m}$ the subspace of $V \cap D\left(A^{\alpha}\right)$ generated by the first $m$ eigenvectors $\omega_{1}, \omega_{2}, \ldots, \omega_{m}$ of $A$. Let $u_{m}(t) \in V_{m}$ defined by:

$$
\left(u_{m}^{\prime \prime}(t), v\right)+M\left(\left|A^{2} u_{m}(t)\right|^{2}\right)\left(A u_{m}(t), v\right)+\left(A^{\alpha} u_{m}^{\prime}(t), v\right)=(f(t), v)
$$

for all $v \in V_{m}$.

$$
\begin{gathered}
u_{m}(0)=u_{o m} \rightarrow u_{o} \text { strongly in } V \cap D\left(A^{\alpha}\right) \\
u_{m}^{\prime}(0)=u_{1 m} \rightarrow u_{1} \text { strongly in } H .
\end{gathered}
$$

The system (8) plus initial conditions (9), (10) has solution on $\left[0, t_{m}[\right.$. Its extension to the interval $[0, T]$ is a consequence of a priori estimates. The crucial point in the proof of Theorem 1.1 is to obtain strong convergence of solutions $\left(u_{m}\right)_{m \in \mathrm{N}}$ in the space $L^{2}\left(0, T ; D\left(A^{2}\right)\right)$, in order to obtain the limit 
of the nonlinear term $M\left(\left|A^{\frac{1}{2}} u_{m}(t)\right|^{2}\right)$. We need two a priori estimates. In the computation we use $u$ in place of $u_{m}$.

FIRST A PRIORI ESTIMATE: Let us represent by $\hat{M}(\lambda)$ a primitive of $M(s)$, i.e.,

$$
\hat{M}(\lambda)=\int_{0}^{\lambda} M(s) d s
$$

Taking $v=2 u^{\prime}$ in (8), integrating from 0 to $t$ and applying CauchySchwarz inequality, we obtain:

$$
\begin{aligned}
& \left|u^{\prime}(t)\right|^{2}+\hat{M}\left(\left|A^{\frac{1}{2}} u(t)\right|^{2}\right)+2 \int_{0}^{t}\left|A^{2} u^{\prime}(s)\right|^{2} d s \\
\leq & 2 \int_{0}^{t}|f(s)|\left|u^{\prime}(s)\right| d s+\left|u_{1 m}\right|^{2}+\hat{M}\left(\left|A^{\frac{1}{2}} u_{o m}\right|^{2}\right)
\end{aligned}
$$

Whence, by the assumption (3) on $M(s)$,

$$
\begin{aligned}
& \left|u^{\prime}(t)\right|^{2}+m_{o}\left|A^{\frac{1}{2}} u(t)\right|^{2}+2 \int_{0}^{t}\left|A^{2} u^{\prime}(s)\right|^{2} d s \\
& \leq\left. 2 \int_{0}^{t}|f(s) \|| u^{\prime}(s)|d s+| u_{1}\right|^{2}+\hat{M}\left(\left|A^{\frac{1}{2}} u_{o}\right|^{2}\right) .
\end{aligned}
$$

Observe that $\hat{M}(\lambda) \geq m_{o} \lambda$. From (11) it follows:

$$
\left|u^{\prime}(t)\right|^{2} \leq\left|u_{\mid}\right|^{2}+\hat{M}\left(\left|A^{\frac{1}{2}} u_{o}\right|^{2}\right)+2 \int_{0}^{\prime}|f(s)|\left|u^{\prime}(s)\right| d s .
$$

Since $|f(s)| \in L^{\mathrm{I}}(0, T)$, this inequality implies, by Gronwall's lemma:

$$
\int_{0}^{1}|f(s)|\left|u^{\prime}(s)\right| d s
$$

is bounded for all $t$ in $[0, T]$. Therefore, from this estimate and (11), we obtain:

$$
\left|u_{m}^{\prime}(l)\right|^{2} \leq \mathrm{C} ;\left|A^{\frac{1}{2}} u_{m}(t)\right|^{2} \leq C ; \int_{0}^{t}\left|A^{\frac{a}{2}} u_{m}^{\prime}(s)\right|^{2} d s \leq C
$$

for all $t \in[0, T]$, where $C>0$ is a constant independent of $m$.

SECOND A PRIORI ESTIMATE: We take $v=A^{\alpha} u(8)$; as above $u$ in place of $u_{m}$. We get:

$\frac{d}{d t}\left(u^{\prime}, A^{\alpha} u\right)-\left|A^{2} u^{\prime}\right|^{2}+M\left(\left|A^{\frac{1}{2}} u\right|^{2}\right)\left|A^{\alpha+1} u\right|^{2}+\frac{1}{2} \frac{d}{d t}\left|A^{\alpha} u\right|^{2}=\left(f, A^{\alpha} u\right)$. 
Integrating from 0 to $t$, we find:

$$
\begin{gathered}
\int_{0}^{t} M\left(\left|A^{2} u\right|^{2}\right)\left|A^{\alpha+1} u\right|^{2} d s+\frac{1}{2}\left|A^{\alpha} u\right|^{2}=-\left(u^{\prime}, A^{\alpha} u\right) \\
+\left(u_{1 m}, A^{\alpha} u_{o m}\right)+\int_{0}^{t}\left|A^{2} u^{\prime}\right|^{2} d s+\frac{1}{2}\left|A^{\alpha} u_{o n}\right|^{2}+\int_{0}^{l}\left(f, A^{\alpha} u\right) d s .
\end{gathered}
$$

By the conditions (9), (10) on $u_{o m}, u_{1 m}$; by the first estimate; by the assumption (3) on $M$ and by the inequality $2 p q \leq \frac{p^{2}}{\beta}+\beta q^{2}, \beta>0$, we obtain,
from (13):

$$
m_{o} \int_{0}^{1}\left|A^{\alpha+1} u\right|^{2} d s+\left(\frac{1}{2}-\beta\right)\left|A^{\alpha} u\right|^{2} \leq K+\int_{0}^{t}\left|f(s) \| A^{\alpha} u(s)\right| d s
$$

for $0<\beta<\frac{1}{2}, K>0$ constant.

We obtain from (14),

$$
\left|A^{\alpha} u(t)\right|^{2} \leq a+b \int_{0}^{1}|f(s) \|| A^{\alpha} u(s) \mid d s,
$$

with $|f(s)| \in L^{1}(0, T)$. By Gronwall's lemma, the last inequality and (14), it follows:

$$
\left|A^{\alpha} u_{m}(t)\right|^{2} \leq C_{1}, \int_{0}^{t}\left|A^{\underline{\alpha}+1} u_{m}(s)\right|^{2} d s \leq C_{1}
$$

with $C_{1}$ independent of $m$, for all $t$ in $[0, T]$.

We have:

$$
D\left(A^{\alpha+1}\right) \subset D\left(A^{2}\right) \subset H, \quad 0<\alpha \leq 1
$$

with continuous injections. We have compact embedding of $D\left(A^{\alpha+1}\right)$ into $D\left(A^{2}\right)$.

From (12), (15) we have:

$$
\begin{aligned}
& \left(u_{m}\right)_{m \in \mathrm{N}} \text { bounded in } L^{2}\left(0, T ; D\left(A^{\alpha+1}{ }^{\alpha+1}\right)\right) \\
& \left(u_{m}^{\prime}\right)_{m \in \mathrm{N}} \text { bounded in } L^{2}(0, T ; H)
\end{aligned}
$$

By (16), (17) and Aubin-Lions compactness theorem, we can extract, from $\left(u_{m}\right)_{m \in \mathrm{N}}$, a subsequence $\left(u_{\mu}\right)_{\mu \in \mathrm{N}}$, such that:

$$
\left(u_{\mu}\right)_{\mu \in \mathrm{N}} \text { converges to } u \text { strongly in } L^{2}\left(0, T ; D\left(A^{2}\right)\right) \text {. }
$$


The vector $u$ obtained from (12), (15), (18), is such that:

$$
\begin{gathered}
\left(u_{\mu}\right)_{\mu \subset N} \text { converges to } u \text { weak star in } L^{\infty}\left(0, T ; V \cap D\left(A^{\alpha}\right)\right) \\
\text { and weakly in } L^{2}\left(0, T ; D\left(A^{a+1}\right)\right) \\
\left(u_{\mu}^{\prime}\right)_{\mu \in N} \text { converges to } u^{\prime} \text { weak star in } L^{\infty}(0, T ; H) \\
\text { and weakly in } L^{2}\left(0, T ; D\left(A^{2}\right)\right) .
\end{gathered}
$$

The convergences (18), (19) imply that we can take the limit, when $\mu \rightarrow \infty$, in the approximated system (8), to obtain (6). We also prove that $u$ satisfies the initial condition (7) and Theorem 1.1 is proved. Q.E.D.

Corollary 1.1: Under the hypothesis of Theorem 1.I, with $f=0$, there exists a function $u:[0, \infty[\rightarrow H$, satisfying the conditions:

$$
\begin{aligned}
& u \in L^{\infty}\left(0, \infty ; V \cap D\left(A^{\alpha}\right)\right) \cap L^{2}\left(0, \infty ; D\left(A^{\alpha+1}\right)\right) \\
& u^{\prime} \in L^{\infty}(0, \infty ; H) \cap L^{2}\left(0, \infty ; D\left(A^{2}\right)\right) \\
& u^{\prime \prime}+M\left(\left|A^{2} u\right|^{2}\right) A u+A^{\alpha} u^{\prime}=0 \text { weakly as }(6) \\
& u(0)=u_{o}, \quad u^{\prime}(0)=u_{l} .
\end{aligned}
$$

The next step of our work is dedicated to analyse the uniqueness. In order to be clear we fixe notations and prove two lemmas.

Let $\beta>0$ be a real number. We consider $D\left(A^{\beta}\right)$ with the norm $\|u\|_{\beta}=$ $\left|A^{\beta} u\right|$. Then,

$$
A^{\beta}: D\left(A^{\beta}\right) \rightarrow H
$$

is linear and continuous. It follows that the adjoint $A^{\beta^{*}}$,

$$
A^{\beta^{*}}: H^{\prime} \rightarrow\left(D\left(A^{\beta}\right)\right)^{\prime}
$$

is also linear and continuous, with

$$
\left\langle A^{\beta^{*}} f, u\right\rangle_{B^{*} \beta}=\left\langle f, A^{\beta} u\right\rangle_{H^{*} \times H}
$$

where \langle\rangle$_{\beta^{\prime} \beta}$ is the duality between $\left(D\left(A^{\beta}\right)\right)^{\rho}$ and $D\left(A^{\beta}\right)$. Note that $E^{r}$ represents the dual of $E$.

Lemma 1.1: If we identify $H$ with $H^{\prime}$ by Riesz isomorphism, then $A^{\beta^{*}}$ is an extension of $A^{\beta}$. 
Proof: If $f \in H^{1}$, then $\langle f, v\rangle=\langle J u, v\rangle=(u, v)$, where $J$ is Riesz's isomorphism. We have

$$
D\left(A^{\beta}\right) \subset H=H^{\prime} \subset\left(D\left(A^{\beta}\right)\right)^{\prime}
$$

continuous and dense. Let $v \in D\left(A^{\beta}\right)$, and $v \in H$ and $J v \in H^{\prime}$. It follows, from (20), that:

$$
\begin{aligned}
\left\langle A^{\beta^{*}} J v, u\right\rangle_{\beta^{*} \beta} & =\left\langle J v, A^{\beta} u\right\rangle_{H^{\prime} \times H}=\left(v, A^{\beta} u\right) \\
& =\left(A^{\beta} v, u\right)=\left\langle J A^{\beta} v, u\right\rangle_{\beta^{\prime} \beta},
\end{aligned}
$$

what implies $A^{\beta^{*}} J v=J A^{\beta} u$. Q.E.D.

Let us consider $\frac{1}{2} \leq \alpha \leq 1$. Then

$$
D\left(A^{\frac{\alpha}{2}}\right) \subset D\left(A^{\frac{1}{2} \frac{\alpha}{2}}\right) \text { and }\left(D\left(A^{\frac{1-a}{2}}\right)\right)^{x} \subset\left(D\left(A^{\frac{\alpha}{2}}\right)\right)^{\prime}
$$

dense and continuous.

Lemma 1.2: If $\frac{1}{2} \leq \alpha \leq I$ and

then

$$
u \in L^{2}\left(0, T ; D\left(A^{\frac{\alpha+1}{2}}\right)\right), \quad u^{\prime} \in L^{2}\left(0, T ; D\left(A^{\frac{\alpha}{2}}\right)\right),
$$

$$
\frac{d}{d l}\left(A^{\frac{\alpha+1}{2}} u, A^{\frac{1-\alpha}{2}} u\right)=2\left(A^{\frac{\alpha+1}{2}} u, A^{\frac{1-\alpha}{2}} u^{\prime}\right)
$$

Proof: Let $W(0, T)$ the space

$$
\left\{v ; v \in L^{2}\left(0, T ; D\left(A^{\frac{\alpha+1}{2}}\right)\right), v^{\prime} \in L^{2}\left(0, T ; D\left(A^{2}\right)\right)\right\}
$$

normed by

$$
\|v\|_{W(0, T)}^{2}=\|v\|_{L^{2}\left(0, T: D\left(A^{2}\right)\right)}^{2}+\left\|v^{\prime}\right\|_{L^{2}\left(0, T: D\left(A^{2}\right),\right.}^{2} \underset{\alpha}{\alpha}
$$

We know that $D\left([0, T] ; D\left(A_{\alpha+1}^{\frac{\alpha+1}{2}}\right)\right)$ is dense in $W(0, T)$, cf. Lions-Magenes [8], p. 13. Let $\varphi \in D\left([0, T] ; D\left(A^{\frac{\alpha+1}{2}}\right)\right)$. Then, 


$$
\begin{aligned}
\frac{d}{d t}\left(A^{\alpha+1} \varphi, A^{\frac{1-\alpha}{2}} \varphi\right) & =\left(A^{\frac{\alpha+1}{2} \varphi^{\prime}}, A^{\frac{1-\alpha}{2}} \varphi\right)+\left(A^{2+1} \varphi, A^{\frac{1-\alpha}{2}} \varphi^{\prime}\right) \\
& =\left(A^{\alpha}\left(A^{\frac{1-\alpha}{2}} \varphi^{\prime}\right), A^{\frac{1-\alpha}{2}} \varphi\right)+\left(A^{\frac{\alpha+1}{2}} \varphi, A^{1-\alpha} \varphi^{\prime}\right) \\
& =\left(A^{1-\frac{\alpha}{2}} \varphi^{\prime}, A^{\alpha}\left(A^{1-\underline{\alpha}} \varphi\right)\right)+\left(A^{\alpha+1} \varphi, A^{2} \varphi^{\prime}\right),
\end{aligned}
$$

since $A^{\frac{1-\alpha}{2}} \varphi, A^{\frac{1-\alpha}{2}} \varphi^{\prime}$ belong to $D\left(A^{\alpha}\right)$.

From (22), we have:

$$
\frac{d}{d t}\left(A^{\frac{a+1}{2}} \varphi, A^{1} i^{2} \varphi\right)=2\left(A^{\alpha+1} \varphi, A^{1-\alpha} \varphi^{\prime}\right) .
$$

If $u$ satisfies the condition in Lemma 2.1 , then there exists a sequence $\left(\varphi_{v}\right)_{v \in \mathbf{N}}$ of $D\left([0, T] ; D\left(A^{\frac{\alpha+1}{2}}\right)\right)$ such that

$$
\begin{aligned}
& \lim \varphi_{v}=u \text { in } L^{2}\left(O, T ; D\left(A^{\frac{\alpha+1}{2}}\right)\right) \\
& \lim \varphi_{v}^{\prime}=u^{\prime} \text { in } L^{2}\left(O, T ; D\left(A^{\frac{1-\alpha}{2}}\right)\right)
\end{aligned}
$$

From (24) we obtain:

$$
\begin{aligned}
& \lim \left(A^{\alpha+1} \varphi_{v}, A^{\frac{1-\alpha}{2}} \varphi_{v}\right)=\left(A^{\frac{\alpha+1}{2}} u, A^{\frac{1-\alpha}{2}} u\right) \text { in } L^{1}(0, T) \\
& \lim \left(A^{\alpha+1} \varphi_{v}, A^{\frac{1-\alpha}{2}} \varphi_{v}^{\prime}\right)=\left(A^{\frac{\alpha+1}{2}} u, A^{\frac{1-\alpha}{2}} u^{\prime}\right) \text { in } L^{1}(0, T)
\end{aligned}
$$

Considering these convergences in the space $D^{\prime}(0, T)$ and taking the limits of (23) with $\varphi=\varphi_{v}$, we prove Lemma 1.2. Q.E.D.

\section{ESTIMATE FOR $u^{\prime \prime}$ :}

Let $u$ the solution of Theorem 1.1 with $f=0$. Then, $u$ satisfies:

$$
\begin{gathered}
-\int_{0}^{T}\left(u^{\prime}, \theta^{\prime} v\right) d t+\int_{0}^{T} M\left(\left|A^{\frac{1}{2}} u\right|^{2}\right)\left(A^{\frac{\alpha+1}{2}} u, \theta A^{\frac{1-\alpha}{2}} v\right) d t \\
+\int_{0}^{T}\left(A^{2} u^{\prime}, \theta A^{2} v\right) d t=0
\end{gathered}
$$

for $\theta \in D(0, T)$ and $v$ eigenvector of $A$. Observe that $M\left(\left|A^{\frac{1}{2}} u\right|^{2}\right) \in L^{\infty}(0, T)$ because $\left(u_{m}\right)_{m \in \mathrm{N}}$ is bounded in $L^{\infty}\left(0, T ; D\left(A^{\frac{1}{2}}\right)\right)$. The eigenvectors of $A$ are 
dense in $D\left(A_{1-\alpha}^{1-\alpha}{ }^{2}\right) \cap D\left(A^{\frac{\alpha}{2}}\right)$ which implies that (25) is true for all $v \in D\left(A^{2}\right) \cap D\left(A^{\frac{\alpha}{2}}\right)$. From (25) with such vectors $v$, we obtain:

$$
\begin{aligned}
-\int_{0}^{T}\left(u^{\prime}, \theta^{\prime} v\right) d t & +\int_{0}^{T} M\left(\left|A^{\frac{1}{2}} u\right|^{2}\right)\left\langle\left(A^{\frac{1-\alpha}{2}}\right)^{*}\left(A^{\alpha+1} u\right), \theta v\right\rangle_{\frac{(1-\alpha}{2} r^{\prime} \frac{(1+\alpha)}{2}} d t \\
+ & \int_{0}^{T}\left\langle\left(A^{2} u\right)^{*}\left(A^{2} u\right), \theta v\right\rangle_{\left(\frac{\alpha}{2}\right)^{\prime}\left(\frac{\alpha}{2}\right)} d t=0 .
\end{aligned}
$$

This implies:

$$
u^{\prime \prime}+M\left(\left|A^{\frac{1}{2}} u\right|\right)\left(A^{\frac{1-\alpha}{2}}\right)^{*}\left(A^{\frac{1-\alpha}{2}} u\right) u+\left(A^{2}\right)^{*} A^{2} u^{\prime}=0
$$

in the sense of $D^{\prime}\left(0, T ;\left(D\left(A^{i-\alpha}\right)\right)^{\prime}+\left(D\left(A^{\frac{\alpha}{2}}\right)\right)^{\prime}\right)$.

Since,

$$
M\left(\left|A^{\frac{1}{2}} u\right|^{2}\right)\left(A^{1-\alpha}\right)^{*}\left(A^{\frac{\alpha+1}{2}} u\right) \in L^{2}\left(0, T ;\left(D\left(A^{1-\alpha}\right)\right)^{\prime}\right)
$$

and

$$
\left(A^{2}\right)^{*} \stackrel{a}{A^{2}} u \in L^{2}\left(0, T ;\left(D\left(A^{2}\right)\right)^{\prime}\right)
$$

from (26):

$$
u^{\prime \prime} \in L^{2}\left(0, T ;\left(D\left(A^{1-\alpha}\right) \cap D\left(A^{2}\right)\right)^{\prime}\right) .
$$
have:

Note that $u^{\prime} \in D\left(A^{\frac{\alpha}{2}}\right)$. Then to make sense the duality $\left(u^{\prime \prime}, u^{\prime}\right)$ we must

$$
D\left(A^{2}\right) \subset D\left(A^{1 / \alpha}\right) \cap D\left(A^{2}\right),
$$

what implies

$$
\frac{\alpha}{2} \geq \frac{1-\underline{\alpha}}{2} \text { or } \alpha \geq \frac{1}{2} \text {. }
$$

It follows that for $\alpha \geq \frac{1}{2}$, we have:

$$
u^{\prime \prime} \in L^{2}\left(0^{*}, T ;\left(D\left(A^{2}\right)\right)^{\prime}\right) .
$$


Remark 1.1: From

$$
u^{\prime} \in L^{2}\left(0, T ; D\left(A^{2}\right)\right) \text { and } u^{\prime \prime} \in L^{2}\left(0, T ;\left(D\left(A^{2}\right)\right)^{\prime}\right) \text {, }
$$

it follows:

$$
u^{\prime} \in C^{o}([0, T] ; H)
$$

and

$$
\frac{d}{d t}\left|u^{\prime}\right|^{2}=2\left(u^{\prime \prime}, u^{\prime}\right)\left(\frac{\alpha}{2}\right)^{\prime} \frac{\alpha}{2}
$$

Theorem 1.2: (Uniqueness). Suppose

$$
M \in C^{l}\left(0, \infty[; \mathbf{R}), \quad M(s) \geq m_{o}>0, \frac{1}{2} \leq \alpha \leq 1 .\right.
$$

If $u, v$ are two solutions of Theorem 1.1, then $u=v$.

Proof: If $\omega=u-v$, from (26) we have:

$$
\begin{gathered}
\omega^{\prime \prime}+M\left(\left|A^{\frac{1}{2}} u\right|^{2}\right)\left(A^{\frac{1-\alpha}{2}}\right)^{*}\left(A^{\frac{\alpha+1}{2}} u\right)-M\left(\left|A^{\frac{1}{2}} v\right|^{2}\right)\left(A^{1 \ldots \alpha}\right)^{*}\left(A^{\alpha+1} v\right) \\
+\left(A^{2}\right)^{*} A^{2} \omega^{\prime}=0 \text { in } L^{2}\left(0, T ;\left(D\left(A^{\frac{\alpha}{2}}\right)^{\prime}\right)\right) \\
\omega(0)=\omega^{\prime}(0)=0 .
\end{gathered}
$$

Since $\omega^{\prime} \in L^{2}\left(0, T ; D\left(A^{2}\right)\right)$, from (28) we obtain:

$$
\begin{aligned}
\int_{0}^{\prime}\left\langle\omega^{\prime \prime}, \omega^{\prime}\right\rangle\left(\frac{\alpha}{2}\right)^{\prime}\left(\frac{\alpha}{2}\right) d s & +\int_{0}^{t} M\left(\left|A^{\frac{1}{2}} u\right|^{2}\right)\left(A^{\frac{\alpha+1}{2}} \omega, A^{\frac{1-\alpha}{2}} \omega^{\prime}\right) d s \\
& +\int_{0}^{t}\left[M\left(\left|A^{\frac{1}{2}} u\right|^{2}\right)-M\left(\left|A^{\frac{1}{2}} v\right|^{2}\right)\right]\left(A^{\frac{\alpha+1}{2}} v, A^{\frac{1-\alpha}{2}} \omega^{\prime}\right) d s \\
& +\int_{0}^{1}\left|A^{\frac{\alpha}{2}} \omega^{\prime}\right|^{2} d s=0 .
\end{aligned}
$$

Remark 1.2: We have $A^{\frac{1-\alpha}{2}} u, A^{\frac{1}{2}} u \in D\left(A^{2}\right)$. Then

$$
\left(A^{\frac{1}{2}} u, A^{2} u\right)=\left(A^{1} u, A^{\frac{\alpha}{2}} A^{1-\alpha}{ }^{2} u\right)=\left(A^{\frac{\alpha+1}{2}} u, A^{1-\alpha} u\right) \text {. }
$$


From this equality and Lemma 1.2, we obtain:

$$
\frac{d}{d t}\left|A^{\frac{1}{2}} u\right|^{2}=2\left(A^{\frac{\alpha+1}{2}} u, A^{\frac{1-a}{2}} u^{\prime}\right)
$$

By Remark 1.1 and Remark 1.2, we get from (29):

$$
\begin{aligned}
\frac{1}{2}\left|\omega^{\prime}(t)\right|^{2} & +\int_{0}^{t} \frac{1}{2} \frac{d}{d s}\left[M\left(\left|A^{\frac{1}{2}} u\right|^{2}\right)\|\omega\|^{2}\right] d s+\int_{0}^{t}\left|A^{\frac{\alpha}{2}} \omega^{\prime}\right|^{2} d s \\
& \left.=\int_{0}^{t} M^{\prime}\left(\left|A^{\frac{1}{2}} u\right|^{2}\right) A^{\frac{\alpha+1}{2}} u, A^{\frac{1}{2}} u^{\prime}\right)\|\omega\|^{2} d s \\
& +\int_{0}^{t} M^{\prime}(\xi)(\|u\|+\|v\|)(\|v\|-\| u||)\left(A^{\frac{\alpha+1}{2}} v, A^{\frac{1-\alpha}{2}} \omega^{\prime}\right) d s
\end{aligned}
$$

We have, observing that $\frac{\alpha}{2} \geq \frac{1-\alpha}{2}$,

$$
\begin{aligned}
& \left|M^{\prime}\left(\left|A^{\frac{1}{2}} u\right|^{2}\right)\left(\frac{\alpha+1}{A^{2} u, A^{\frac{1-\alpha}{2}}} u^{\prime}\right)\|\omega\|^{2}\right| \\
& \leq c\left|A^{\frac{\alpha+1}{2}} u\right|^{2}\|\omega\|^{2}+\left.c\left|A^{\frac{\alpha}{2}} u^{\prime}\right|^{2}\|\omega\|\right|^{2} .
\end{aligned}
$$

Also we have:

$$
\begin{aligned}
& \quad\left|M^{\prime}(\xi)\right|(\|u\|+\|v\|)(\|v\|-\|u\|)\left(A^{\frac{\alpha+1}{2}} v, A^{\frac{1}{2} \frac{\alpha}{2}} \omega^{\prime}\right) \\
& \leq c_{1}\|\omega\|\left|A^{\frac{\alpha+1}{2}} \omega\right|\left|A^{\frac{\alpha}{2}} \omega^{\prime}\right| \leq c_{2}\left|A^{\frac{\alpha+1}{2}} v\right|^{2}\|\omega\|^{2}+\eta\left|A^{\frac{\alpha}{2}} \omega^{\prime}\right|^{2} \\
& 0<\eta<1 .
\end{aligned}
$$

By (30), (31) and (32), we obtain:

$$
\begin{gathered}
\frac{1}{2} m_{0}\|\omega\|^{2}+(1-\eta) \int_{0}^{t}\left|A^{\frac{\alpha}{2}} \omega^{\prime}\right|^{2} \leq c \int_{0}^{t}\left|A^{\frac{\alpha+1}{2}} u\right|^{2}\|\omega\|^{2} d s \\
+c \int_{0}^{t}\left|A^{\frac{\alpha}{2}} u^{\prime}\right|^{2}\|\omega\|^{2} d s+c_{2} \int_{0}^{t}\left|A^{\frac{\alpha+1}{2}} v\right|^{2}\|\omega\|^{2} d s .
\end{gathered}
$$

This inequality implies:

$$
\|\omega(t)\|\left\|^{2} \leq \int_{0}^{t} g(s)\right\| \omega(s)\|\|^{2} d s, \quad g \in L^{1}(0, T),
$$

that is, $\omega(t)=0,0 \leq t \leq T$. Thus we have uniqueness. Q.E.D. 


\section{ASYMPTOTIC BEHAVIOR}

On this section we obtain informations on the behavior of the energy associated to (6), Section 1, when $t$ goes to $\infty$.

Theorem 2.1: If $u$ is the solution of Corollary 1.I, then

$$
E(t) \leq 4 E(0) e^{\gamma t} \text {, for all } t \geq 0 \text {. }
$$

where

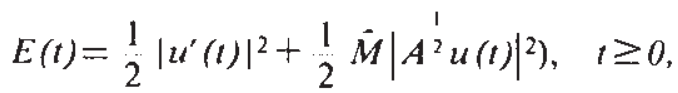

and

$$
\begin{aligned}
& 2 \gamma=\left(\frac{m_{s}}{\tau}\right) \min \left(\frac{m_{s}^{2} \lambda_{1}}{2 m_{s,}^{3}{ }^{2} \Lambda_{1}^{\prime}{ }^{2}+\tau \lambda_{1}+\tau}, \frac{2 \tau \lambda_{\mathrm{I}}^{\alpha}}{2 \tau+m_{\omega}}\right) \\
& \tau=\max \left\{M(s) ; 0 \leq s \leq \frac{2 E(0)}{m_{c}}\right\} \text {, }
\end{aligned}
$$

$\lambda_{1}$ the first eigenvalue of $A$.

We use, in the proof, the method of Haraux-Zuazua [6], cf. also Zuazua [22]. It is sufficient to obtain (33) for the approximated solutions $u_{n}$ of (8). The convergences obtained in the proof of Theorem 1.1 imply the inequality (33) for the limit $u$. By this reason we write $u$ in place of $u_{m}$ and $E(t)$ or $E$ in place of $E_{m}(t)$.

For $\epsilon>0$ we define the perturbed energy $E_{\mathrm{t}}(t)$ by:

$$
E_{\mathrm{c}}(t)=(1+\epsilon C) E(t)+\epsilon\left(u^{\prime}, u\right),
$$

where $C$ is a constant to be determined later.

The method depends on an inequality relating $E(t)$ and $E_{\mathrm{t}}(t)$, which is isolated in the following lemma:

Lemma 2.1: We have

$$
\frac{l}{2} E_{t}(t) \leq E(t) \leq 2 E_{t}(t)
$$


for all $t \geq 0$ and $0<\epsilon \leq \epsilon_{o}$, where

$$
\epsilon_{0}=\frac{\left(m_{0} \lambda_{l}\right)^{l / 2}}{2\left(1+c\left(m_{0}, \lambda_{1}\right)^{l / 2}\right)} .
$$

Proof: Let us consider $\psi(t)=\left(u^{\prime}(t), u(t)\right)$. We have:

$$
|\psi(t)| \leq \frac{\mu}{2}|u(t)|^{2}+\frac{1}{2 \mu}\left|u^{\prime}(t)\right|^{2}, \quad \mu>0,
$$

and we know that $\left|A^{2} u(t)\right|^{2} \geq \lambda_{1}|u(t)|^{2}$, then

$$
|u(t)| \leq \frac{1}{m_{0} \lambda_{1}} \hat{M}\left(\left|A^{2} u(t)\right|^{2}\right) .
$$

From (39), (40) if follows:

$$
|\psi(t)| \leq \frac{\mu}{2 m_{\theta} \lambda_{1}} \hat{M}\left(\left|A^{2} u(t)\right|^{2}\right)+\frac{1}{2 \mu}\left|u^{\prime}(t)\right|^{2} .
$$

Choosing $\mu=\left(m_{0} \lambda_{1}\right)^{2}$, we get:

$$
|\psi(t)| \leq\left(m_{0}, \lambda_{1}\right)^{-\frac{1}{2}} E(t),
$$

and we have:

$$
E_{\epsilon}(t) \leq(1+\epsilon C) E(t)+\epsilon\left(m_{o} \lambda_{1}\right)^{-1}{ }^{-1} E(t) .
$$

This inequality implies:

$$
E_{\mathrm{\varepsilon}}(t) \leqslant 2 E(t),
$$

for $0<\epsilon \leq \epsilon_{0}$.

From (41) and the definition of $E$, we get:

$$
E_{\epsilon}(t) \geq E(t)-\epsilon|\psi(t)| \geq E(t)-\epsilon_{t}\left(m_{i} \lambda_{1}\right)^{-1} 2 E(t) \geq \frac{1}{2} E(t),
$$

which is the other side of the inequality in Lemma 2.1. Q.E.D.

Proof of Theorem 2.1: The idea of the proof is to obtain $E_{t}(t) \leq-\eta E(t)$, $\eta$ a positive constant. This inequality, with Lemma 2.I, permit us to obtain the exponential decay. 
We have from the approximated equation:

$$
E^{\prime}(l)=-\left|A^{\prime \prime} u^{\prime}(l)\right|^{2}
$$

hence,

$$
E^{\prime}(t) \leq-\lambda_{1}^{\alpha}\left|u^{\prime}\right|^{2}
$$

Also from the approximated equation (8) we obtain:

$$
d_{d t}^{d}\left(u^{\prime}, u\right)=\left|u^{\prime}\right|^{2}-M\left(\left|A^{2} u\right|^{2}\right)\left|A^{2} u\right|^{2}-\left(A^{2} u^{\prime}, A^{2} u\right) .
$$

Thus, from (44), (45) we get:

$$
\begin{aligned}
E^{\prime}(t)+\epsilon \frac{d}{d t}\left(u^{\prime}, u\right) & \leq-\lambda_{\mid}^{\alpha}\left|u^{\prime}\right|^{2}+\epsilon\left|u^{\prime}\right|^{2}-\epsilon M\left(\mid A^{2} u^{2}\right)\left|A^{2} u\right|^{2} \\
& -\epsilon\left(A^{2} u^{\prime}, A^{2} u\right), \quad \epsilon>0
\end{aligned}
$$

We have:

$$
\left|\left(A^{2} u^{\prime}, A^{2} u\right)\right| \leq \frac{\delta}{2}\left|A^{2} u^{\prime}\right|^{2}+\frac{1}{2 \delta}\left|A^{2} u\right|^{2}
$$

Since $0<\alpha \leq 1$, we get:

$$
\begin{aligned}
& \left|A^{2} u\right|^{2}=\sum_{0<\lambda_{1} \leq 1} \lambda_{v}^{\alpha}\left|\left(u, \omega_{v}\right)\right|^{2}+\sum_{\lambda_{1} \geq_{1}} \lambda_{i}^{\alpha}\left|\left(u, \omega_{v}\right)\right|^{2} \\
& \leq|u|^{2}+\left|A^{2} u\right|^{2} \leq\left[\frac{1+\lambda_{1}}{m_{*}, \lambda_{1}}\right] \hat{M}\left(\left|A^{\frac{1}{2}} u\right|^{2}\right) .
\end{aligned}
$$

By (43) and this last inequality, we obtain, from (47):

$$
\left|\left(A^{2} u^{\prime}, A^{2} u\right)\right| \leq-\frac{\delta}{2} E^{t}(t)+\frac{k}{2 \delta} \hat{M}\left(\left|A^{2} u\right|^{2}\right)
$$

for

$$
k=\frac{1+\lambda_{1}}{m_{v} \lambda_{1}}
$$


The inequality (48) permits us to write (46) in the form:

$$
\begin{aligned}
& E^{\prime}(l)+\epsilon \frac{\delta}{2} E^{\prime}(t)+\epsilon \frac{d}{d t}\left(u^{\prime}, u\right) \leq-\left(\lambda_{1}^{\alpha}-\epsilon\right)\left|u^{\prime}\right|^{2} \\
& -\epsilon M\left(\left|A^{\frac{1}{2}} u\right|^{2}\right)\left|A^{2} u\right|^{2}+\frac{\epsilon k}{20} \hat{M}\left(\left|A^{2} u\right|^{\prime}\right) .
\end{aligned}
$$

We need to compare the last two terms of (49). For this, we know from (11) in Section 1, since $f=0$ :

$$
\left|A^{\frac{1}{2}} u(t)\right|^{2} \leq \frac{2 E(0)}{m_{t}}, \text { for all } t \geq 0
$$

which implies:

$$
M\left(\left|A^{\frac{1}{2}} u(t)\right|^{2}\right) \leq \tau \text { for all } t \geq 0
$$

with + defined by (36).

Define

$$
a=\frac{2 E(0)}{m_{t \prime}} \text { and } \delta=\frac{k \tau}{m_{i}} .
$$

By the definition of $\hat{M}(\lambda)$, we have for $0 \leq \lambda \leq a$ :

$$
\hat{M}(\lambda) \leq \lambda \max _{0 \leq \xi \leq a} M(\xi)=\frac{\lambda \delta}{k} m_{0} \leq \frac{\lambda \delta}{k} M(\lambda) .
$$

Therefore,

$$
\epsilon \lambda M(\lambda)-\frac{\epsilon k}{2 \delta} \hat{M}(\lambda) \geq \frac{\epsilon k}{2 \delta} \hat{M}(\lambda) \text { for all } 0 \leq \lambda \leq a .
$$

By (50), (51) and observing that $\frac{k}{\tau}=\frac{m_{o}}{\tau}$, we have from (49)

$$
\begin{aligned}
& E^{\prime}(t)+\epsilon \frac{\delta}{2} \cdot E^{\prime}(t)+\epsilon \frac{d}{d t}\left(u^{\prime}, u\right) \\
& \leq-\left(\lambda_{1}^{\alpha}-\epsilon\right)\left|u^{\prime}\right|^{2}-\frac{\epsilon m_{0}}{2 \tau} \hat{M}\left(\left|A^{2} u\right|^{2}\right) .
\end{aligned}
$$


For $0<\epsilon<\epsilon_{1}=\frac{2 \lambda_{1}^{\alpha} \tau}{2 \tau+m_{t}}$, we obtain:

$$
\lambda_{1}^{n}-\epsilon>0 \text { and } \frac{\epsilon m_{u}}{2 \tau}<\lambda_{1}^{r}-\epsilon
$$

Thus, from (52):

$$
E^{\prime}(t)+\frac{\epsilon \delta}{2} E^{\prime}(t)+\epsilon \frac{d}{d t}\left(u^{\prime}, u\right) \leq-\frac{\epsilon m_{*}}{\tau} E(t), 0<\epsilon<\epsilon_{1} .
$$

By L.emma 2.1, with $C=\frac{\delta}{2}$, it follows, from (53):

$$
E_{\mathrm{c}}^{\prime}(t) \leq-\frac{\epsilon m_{i,}}{Z_{1}} E_{\mathrm{c}}(t), \quad 0<\epsilon<\epsilon_{2}=\min \left(\epsilon_{i,}, \epsilon_{1}\right),
$$

which implies:

$$
E_{\mathrm{q}}(t) \leq E_{\mathrm{q}}(0) e^{-\frac{t m_{\mathrm{c}} t}{2 r}} .
$$

If we apply l.emma 2.1, with $0<\epsilon<\epsilon_{2}$, to (54) we obtain Theorem 2.1. Q.E.D.

Remark 1.3: In Y. Yamada [21], he observed that if

$$
M \in C^{1}([0, \infty) ; \mathbf{R}), \quad \frac{1}{2} \leq \alpha \leq 1,
$$

with $\left(u_{0}, u_{1}\right) \in V \cap D\left(A^{\alpha}\right) \times H, f=0$ to avoid technalities, then he obtained further regularity for the solution $u$ given by Theorem 1.1. In fact, he proves that if we have (55), then $u$ satisfy:

$$
\begin{aligned}
& \sqrt{t} u \in L^{\infty}\left(0, T ; D\left(A^{n ! 1}\right)\right) \\
& \sqrt{i} u^{\prime} \in L^{\infty}\left(0, T: D\left(A^{\prime 2}\right) \cap L^{2}\left(0, T: D\left(A^{\alpha}\right)\right)\right. \\
& \sqrt{i} u^{\prime \prime} \in L^{2}(0, T ; H)
\end{aligned}
$$

for all $t \geq 0$.

\section{ACKNOWLEDGEMENTS}

We acknowledge E. Zuazua and Yoshio Yamada for their constructive remarks on this work. We also appreciate very much the suggestions by the Referee. 


\section{References}

[1] ARosio A.; SPAGNolo, S. (1984): Global solutions to the Cauchy problem for a nonlinear hyperbolic equation. Nonlinear Partial Differential Equations and Their Applications, Coll'ege de France Seminar, Vol. 6, edited by $\mathrm{H}$. Brezis and J. L. Lions, Pitman.

[2] BERNSTEIN, S. (1940): Sur une classe d'equations fonctionelles aux derivées partielles, Izv. Acad. Nauk SSSR, Ser. Math. 4, pp. 17-26.

[3] CARRIER, G. F. (1945): On the vibration problem of elastic string, Q.J. Appl. Math. 3, pp. 151-165.

[4] DICKEY, R. W. (1969): Infinite systems of nonlinear oscillations equations related to string, Proc. A.M.S. 23, pp. 459-469.

[5] Ebihara Y.; Medeiros, L. A.; Milla Miranda, M. (1986): Local solutions for a nonlinear degenerated hyperbolic equations, Nonlinear Analysis 10, pp. $27-40$.

[6] HARAUX, A.; ZUAZUA, E. (1988): Decay estimates for some semilinear damped hyperbolic problem, Arch. For Rational Mechanis and Analysis, V. 100, N.2, pp. 191-206.

[7] LiONS, J, L. (1978): On some questions in boundary value problems of mathematical physics, in Contemporary Development in Continuous Mechanics and Partial Differential Equations (Ed. G. de la Penha-L. A. Medeiros), North Holland, London.

[8] Lions, J. L.; MAGENES, E. (1968): Problémes aux limites non homogénes et applications, Vol. 1, Dunod, Paris.

[9] MATOS, M. P.: Mathematical analysis of the nonlinear model for vibration of strings (to appear).

[10] MATOS, M. P.; PEREIRA, D. C.: Hyperbolic equation with sirong damping (to appear).

[11] Medeiros, L. A.; Mil.l.A Miranda, M. (1987): Solution for the equation of nonlinear vibration in Sobolev spaces of fractionary order. Mat. Aplic. Comp. V. 6, N.. 3, pp. 257-276.

[12] MUÑoz RiverA, J. E.: Smoothness effect and stability of nonlinear evolution equation (to appear).

[13] NAKAO, M. ([977): Decay of solutions of some nonlinear evolution equation, Journal of Math. Ann. and Appl. 60, pp. 542-549.

[14] NAKAO, M. (1976): Convergence of solution of the wave equation with nonlinear dissipative term to the steady state, Mem. Fac. Sci. Kyushi University 30 , pp. 257-265.

[15] NARASHIMHAM, R. (1968): Nonlinear vibrations of an elastic string. J. Sound Vib. 8, pp. 134-136.

[16] NISHIHARA, K. (1984): Degenerate quasilinear hyperbolic equation with strong damping, Funcialaj Ekvacioj 27, pp. 125-145.

[17] PohozhaEv, S. (1975): On a class of quasilinear hyperbolic equations, Math. Sbornik 95, pp. 152-166.

[18] Stkauss, W. A. (1969): The energy method in nonlinear partial differential equations. Notas de Matemática 47, IMPA Rio de Janeiro.

[19] TSUTSUM], M. (1971): Some nonlinear evolution equation of second order, Proc. Acad. of Sciences of Japan 47, pp. 450-455.

[20] YAMADA, Y. (1970): On the decay of solutions for some nonlinear evolution equations of second order, Nagoya Math. Journal 73, pp. 69-98. 
[21] Yamada, Y. (1990). Personal communication.

[22] ZuAzUA, E. (1988): Stability and decay for a class of nonlinear hyperbolic problems. Asymptotic Analysis 1, pp. 141-185.

Instituto de Matemátiça, UFRJ

Cx. Postal 68530-CEP: 21944

Rio de Janeiro, R.J - Brasil

Recibido: 10 de octubre de 1989

Revisado: 23 de marro de 1990 